\title{
Can restricting calories help you to live longer?
}

\author{
Dr James E. Brown
}

Aston Research Centre for Healthy Ageing \& School of Life and Health Sciences, Aston University, Birmingham, B4 7ET, UK

Address for correspondence: Dr James E Brown, Aston Research Centre for Healthy Ageing \& School of Life and Health Sciences, Aston University, Birmingham, B4 7ET, UK

Email: j.e.p.brown@aston.ac.uk

Telephone: 01212045039

Fax: 01212043696

\section{Abbreviations and Acronyms}

CR - Calorie restriction

T2D - type 2 diabetes

CVD - Cardiovascular disease

IF - Intermittent fasting

TDEE - Total daily energy expenditure

\begin{abstract}
Excess calorie consumption is associated with metabolic disorders and increased incidence of morbidity. Restricting calorie content, either by daily calorie restriction or intermittent fasting periods, has multiple benefits including weight loss and improved body composition. Previous research has shown that restricting calories in this way can increase longevity and slow the ageing process in laboratory animals, although only sparse data exists in human populations. This review critically evaluates the benefits of these dietary interventions on age-related decline and longevity.
\end{abstract}




\section{Introduction.}

Epidemiological studies have shown that the UK population is becoming increasingly skewed towards older adults [1], and that this trend is likely to continue in the future. Concomitantly, increasing levels of obesity have led to predictions that 700 million people worldwide will be obese by 2015 [2]. Of key importance among these predictions is the projected rapid increase in incidence of obesity in children, where evidence exists for a growing global obesity epidemic among school-age children [3]. Importantly, obesity is associated with significant comorbidities including type 2 diabetes (T2D) and cardiovascular disease (CVD) [4-6], which are also considered to be disorders associated with ageing.

Whilst these increases in obesity rates have been observed, there has been a relative dearth of novel therapies being developed to tackle obesity [7]. There are currently almost no pharmaceutical options available for physicians to treat obesity with and traditional dietary intervention fails in many, bringing the use of standard diet plans into question.

Basic laboratory experiments have shown that by severely restricting calorie intake one can not only induce weight loss but can also influence the ageing process [8]. There is also evidence that limiting food intake in laboratory animals in this way can reduce the levels of age-related decline, increasing what is commonly referred to as 'healthspan' [9] or the relative amount of time spent in good health. Whilst this evidence is strong in animals, there remain questions over how this translates to human populations.

\section{Calorie restriction and intermittent fasting: can decreasing food intake have an anti-ageing effect in humans?}

Many of the genes that are known to be involved in energy balance and metabolic disorders are also involved in the ageing process, highlighting the connection between a healthy energy balance and successful ageing. Large scale population studies have shown that being obese significantly increases the risk of all-cause mortality [10] and there are many established links between excess adiposity and age-related decline conditions including Alzheimer's disease, stroke and cancer [11-13]. Although in recent times have been accompanied by profound increases in life expectancy [14] the possibility exists that these increases may well be tempered by the increased mortality associated with increased obesity levels. Equally, as obesity is associated with accelerated age-related decline the possibility exists that longevity may remain unchanged but healthspan will be significantly reduced.

Research has shown that dietary interventions have a role in modulating both metabolism and successful ageing, with calorie restriction (CR) and intermittent fasting (IF) being shown to not only improve metabolic health, but also prevent a variety of other age-related diseases including cancer [15, 16], immune disorders [17, 18], eye disease [19] and heart disease [20]. It has been known for over 70 years now that a link also exists between nutrition and longevity, with CR significantly increasing lifespan [21]. CR describes a dietary intervention where calories are restricted every day, to a minimum of $40-50 \%$ of normal calorie intake. CR is the dietary intervention that has the greatest evidence base supporting its effects on longevity and delayed age-related decline [22] and has been shown to have a significant impact on longevity across the vast majority of organisms tested, from nematode worms [23, 24] to rodents [25]. 
IF is a more transient form of CR, and has been practiced in human populations for thousands of years in the form of religious fasts [26, 27]. Recently a form of IF has been developed that appears to be more adherable than and as effective as CR, and has been termed " $5: 2$ fasting" [28]. This eating plan consists of days of 'normal' calorie consumption, where an individual may consume their normal total daily energy expenditure (TDEE) in calories, and days where an individual limits calorie consumption to approximately one quarter of their TDEE (600 calories for men, 500 for women) [29]. Research has shown that IF is a powerful dietary tool, with improvements in glucose homeostasis and cardiovascular health being reported [20, 30, 31]. Experimental evidence also suggests that IF can also modulate longevity in laboratory animals [32, 33] much in the same way that CR can.

Although there are such conserved effects of CR/IF on longevity across many species, recent data from one of the earliest studies into CR in non-human primates has cast significant doubt on any impact on human longevity [34]. The implications of these findings are that there is no concrete evidence that either CR or IF can increase longevity in humans. However, numerous studies have shown that these interventions have the potential to decrease the risk of age-related decline which may possibly influence an individual's healthspan.

\section{Summary}

Restricting calorie intake has been shown to be an effective method of manipulating the ageing process in laboratory animals. Although there is little evidence that these dietary interventions can regulate longevity in human populations, there is growing proof that they may be able to reduce levels of age-related decline, and therefore have an impact on healthspan. More clinical trials are needed to fully assess the impact of CR and IF on diseases associated with age-related decline.

\section{References}

1. Lutz, W., W. Sanderson, and S. Scherbov, The coming acceleration of global population ageing. Nature, 2008. 451(7179): p. 716-9.

2. Eshghinia, S. and F. Mohammadzadeh, The effects of modified alternate-day fasting diet on weight loss and CAD risk factors in overweight and obese women. J Diabetes Metab Disord, 2013. 12(1): p. 4.

3. Wang, Y. and T. Lobstein, Worldwide trends in childhood overweight and obesity. Int J Pediatr Obes, 2006. 1(1): p. 11-25. 
4. Wilmot, E.G., et al., Sedentary time in adults and the association with diabetes, cardiovascular disease and death: systematic review and meta-analysis. Diabetologia, 2012. 55(11): p. 2895-905.

5. Warren, T.Y., et al., Sedentary behaviors increase risk of cardiovascular disease mortality in men. Med Sci Sports Exerc, 2010. 42(5): p. 879-85.

6. Estruch, R., et al., Primary prevention of cardiovascular disease with a Mediterranean diet. N Engl J Med, 2013. 368(14): p. 1279-90.

7. Thaler, J.P. and M.W. Schwartz, Minireview: Inflammation and obesity pathogenesis: the hypothalamus heats up. Endocrinology, 2010. 151(9): p. 4109-15.

8. Smith, D.L., Jr., T.R. Nagy, and D.B. Allison, Calorie restriction: what recent results suggest for the future of ageing research. Eur J Clin Invest, 2010. 40(5): p. 440-50.

9. Curtis, J. and R. de Cabo, Utilizing calorie restriction to evaluate the role of sirtuins in healthspan and lifespan of mice. Methods Mol Biol, 2013. 1077: p. 303-11.

10. Flegal, K.M., et al., Association of all-cause mortality with overweight and obesity using standard body mass index categories: a systematic review and meta-analysis. JAMA, 2013. 309(1): p. 71-82.

11. Barrett-Connor, E., An introduction to obesity and dementia. Curr Alzheimer Res, 2007. 4(2): p. 97-101.

12. Barrett-Connor, E., Obesity, hypertension and stroke. Clin Exp Hypertens A, 1990. 12(5): p. 769-82.

13. Protani, M., M. Coory, and J.H. Martin, Effect of obesity on survival of women with breast cancer: systematic review and meta-analysis. Breast Cancer Res Treat, 2010. 123(3): p. 62735.

14. Jackson, M., Disease and diversity in history. (Reviews of: Cartwright, FF, Biddiss, M. Disease and history. Gloucestershire: Sutton Publishing Limited, 2000; Rotberg Rl, ed. Health and disease in human history. Cambridge, MA: MIT Press, 2000; Cliff, A., Haggett, P., SmallmanRaynor, M. Deciphering global epidemics: analytical approaches to the disease records of world cities, 1888-1912. Cambridge: Cambridge University Press, 1998; Riley, JC. Rising life expectancy: a global history. Cambridge: Cambridge University Press, 2001; McMichael, T. Human frontiers, environments and disease: past patterns, uncertain futures. Cambridge: Cambridge University Press, 2001). Soc Hist Med, 2002. 15(2): p. 323-40.

15. Longo, V.D. and L. Fontana, Calorie restriction and cancer prevention: metabolic and molecular mechanisms. Trends Pharmacol Sci, 2010. 31(2): p. 89-98.

16. Galet, C., et al., Effects of Calorie Restriction and IGF-1 Receptor Blockade on the Progression of 22Rv1 Prostate Cancer Xenografts. Int J Mol Sci, 2013. 14(7): p. 13782-95.

17. Esquifino, A.I., et al., Immune response after experimental allergic encephalomyelitis in rats subjected to calorie restriction. J Neuroinflammation, 2007. 4: p. 6.

18. Ahmed, T., et al., Calorie restriction enhances $T$-cell-mediated immune response in adult overweight men and women. J Gerontol A Biol Sci Med Sci, 2009. 64(11): p. 1107-13.

19. Kawashima, M., et al., Calorie restriction: A new therapeutic intervention for age-related dry eye disease in rats. Biochem Biophys Res Commun, 2010. 397(4): p. 724-8.

20. Kroeger, C.M., et al., Improvement in coronary heart disease risk factors during an intermittent fasting/calorie restriction regimen: Relationship to adipokine modulations. Nutr Metab (Lond), 2012. 9(1): p. 98.

21. Mccay, C.M., M.F. Crowell, and L.A. Maynard, Nutrition Metabolism Classic - the Effect of Retarded Growth Upon the Length of Life-Span and Upon the Ultimate Body Size (Reprinted from Journal of Nutrition, Vol 10, Pg 63-79, 1935). Nutrition, 1989. 5(3): p. 155-171.

22. Masoro, E.J., Overview of caloric restriction and ageing. Mech Ageing Dev, 2005. 126(9): p. 913-22. 
23. Jadiya, P., et al., Sir-2.1 modulates 'calorie-restriction-mediated' prevention of neurodegeneration in Caenorhabditis elegans: implications for Parkinson's disease. Biochem Biophys Res Commun, 2011. 413(2): p. 306-10.

24. Smith, E.D., et al., Age- and calorie-independent life span extension from dietary restriction by bacterial deprivation in Caenorhabditis elegans. BMC Dev Biol, 2008. 8: p. 49.

25. Lam, Y.Y., C.M. Peterson, and E. Ravussin, Resveratrol vs. calorie restriction: data from rodents to humans. Exp Gerontol, 2013. 48(10): p. 1018-24.

26. Faris, M.A., et al., Intermittent fasting during Ramadan attenuates proinflammatory cytokines and immune cells in healthy subjects. Nutr Res, 2012. 32(12): p. 947-55.

27. Sweileh, N., et al., Body composition and energy metabolism in resting and exercising muslims during Ramadan fast. J Sports Med Phys Fitness, 1992. 32(2): p. 156-63.

28. Harvie, M.N., et al., The effects of intermittent or continuous energy restriction on weight loss and metabolic disease risk markers: a randomized trial in young overweight women. Int J Obes (Lond), 2011. 35(5): p. 714-27.

29. Brown, J.E., M. Mosley, and S. Aldred, Intermittent fasting: a dietary intervention for prevention of diabetes and cardiovascular disease? The British Journal of Diabetes \& Vascular Disease, 2013. 13(2): p. 68-72.

30. de Azevedo, F.R., D. Ikeoka, and B. Caramelli, Effects of intermittent fasting on metabolism in men. Rev Assoc Med Bras, 2013. 59(2): p. 167-73.

31. Klempel, M.C., et al., Intermittent fasting combined with calorie restriction is effective for weight loss and cardio-protection in obese women. Nutr J, 2012. 11: p. 98.

32. Honjoh, S., et al., Signalling through RHEB-1 mediates intermittent fasting-induced longevity in C. elegans. Nature, 2009. 457(7230): p. 726-30.

33. INTERMITTENT fasting and longevity in rats. Nutr Rev, 1946. 4(7): p. 218.

34. Mattison, J.A., et al., Impact of caloric restriction on health and survival in rhesus monkeys from the NIA study. Nature, 2012. 489(7415): p. 318-21. 